\title{
Current Issues in Atrial Fibrillation
}

\author{
Yaariv Khaykin ${ }^{1,2}$ and Yana Shamiss ${ }^{1}$ \\ ${ }^{1}$ Heart Rhythm Program, Southlake Regional Health Centre, 105-712 Davis Drive, Newmarket, ON, Canada L3Y 8C3
}

${ }^{2}$ Faculty of Medicine, University of Toronto, Toronto, ON, Canada M5S 1A8

Correspondence should be addressed to Yaariv Khaykin, y.khaykin@utoronto.ca

Received 20 March 2012; Accepted 26 April 2012

Academic Editors: J. Brachmann and G. Iaccarino

Copyright ( $) 2012$ Y. Khaykin and Y. Shamiss. This is an open access article distributed under the Creative Commons Attribution License, which permits unrestricted use, distribution, and reproduction in any medium, provided the original work is properly cited.

Atrial fibrillation (AF) is the most common sustained cardiac arrhythmia. It places an enormous burden on the patients, caregivers, and the society at large. While the main themes in the care of an AF patient have not changed over the years and continue to focus on stroke prevention, control of the ventricular, rate and rhythm maintenance, there have been a number of new developments in each of these realms. This paper will discuss the "hot" topics in AF in 2012 including new and upcoming medical and invasive management strategies for this condition.

\section{Introduction}

"There is no other serious cardiac disorder which can be so speedily benefited as the well-managed case of auricular fibrillation...the most reliable preparation to use is a fresh and known tincture of digitalis."

This quote from Clinical Disorders of the Heart Beat by Lewis published in 1925 [1] could not be further from the truth in 2012. With the multitude of new therapies introduced and in development to address various clinical implications of this most common sustained rhythm disorder, it is becoming a daunting task to select the right approach to each individual patient.

Atrial fibrillation (AF) is responsible for most arrhythmia-related hospital admissions [2] and is the most common cause of ischemic stroke [3]. Furthermore, AF carries a tremendous negative impact on the quality of life and is associated with increased mortality [4]. Its prevalence is rising in our ageing society $[5,6]$ and so does the expense related to its management [7] and productivity lost among the suffering patients [8].

Decisions that need to be made in each AF patient care include selection of rhythm control or the more conservative control of the ventricular rate and selection of stroke prevention strategy. At each step the clinician needs to decide between medical and invasive solutions. Since AF is frequently associated with other comorbid conditions, these need to be addressed as well.

Clinical practice guidelines developed by various professional societies attempt to help physicians select the right therapies for the right AF patients. Unfortunately, the many nuances of AF presentation and available therapies complicate clinical decision-making, guidelines lag behind new clinical developments, and few mechanisms are in place to translate guidelines into standards of care. The purpose of this paper is to discuss the "hot" topics in AF care in 2012.

\section{Preventing Embolic Sequelae}

Prevention of embolic complications is the most important aspect of care for AF patients. These range from transient ischemic events (TIAs) to strokes and are the most costly complication of atrial fibrillation. Strokes secondary to AF are more severe than those secondary to atherosclerotic disease and impart a greater disability on the victims [7]. This results in significant costs related to hospitalizations, rehabilitation, and chronic disability. Strategies aimed at reducing embolic events in AF patients include therapy with aspirin, combination of aspirin and clopidogrel, and oral anticoagulation therapy with warfarin or one of the new agents targeting either thrombin or Factor Xa [9-11]. 
Despite all of this, currently as few as 10-20\% of the AF patients are treated with appropriate prophylaxis strategies [12]. Those who do take warfarin spend much of their time taking subtherapeutic doses of the medication placing them at risk of stroke, while others take supertherapeutic doses and run a significant risk of bleeding, given a very narrow therapeutic range of this drug. Novel antithrombotic agents allow for more consistent anticoagulation and have been shown superior to warfarin in stroke prevention.

In the open-label study of Dabigatran versus Warfarin in Patients with Atrial Fibrillation (RELY) the use of dabigatran, a direct thrombin inhibitor, was associated with similar rates of stroke and systemic embolism but lower rate of major bleeding compared to warfarin at a lower dose of $110 \mathrm{mg}$, while the higher dose of the drug at $150 \mathrm{mg}$ was associated with $35 \%$ lower rates of stroke and systemic embolism but similar rates of major bleeding compared to warfarin [11]. Similarly, in the blinded study of Apixaban versus Warfarin in Patients with Atrial Fibrillation (ARISTOTLE), apixaban, a factor $\mathrm{Xa}$ inhibitor, was superior to warfarin in preventing stroke or systemic embolism with a $21 \%$ relative risk reduction in this outcome. Apixaban caused less bleeding with a relative risk reduction of over $30 \%$ and lowered all-cause mortality by $11 \%$ [13].

Rivaroxaban, another factor Xa inhibitor, was studied in a somewhat different setting than the other two agents. While most patients in the RELY and ARISTOTLE studies were at a relatively low risk for stroke, in the rivaroxaban once daily oral direct factor Xa inhibition compared with vitamin $\mathrm{K}$ antagonism for prevention of stroke and Embolism Trial in Atrial Fibrillation (ROCKET-AF) study, only 10\% of the patients had CHADS-2 score less than 3 with the remainder of the patients at higher risk of stroke. Unlike the other two agents, rivaroxaban was shown to be noninferior to warfarin in these patients in the intention-to-treat analysis but reduced stroke or systemic embolism by $21 \%$ among patients actually taking the drug [14]. All of these agents are renally eliminated to some extent requiring dose adjustment among patients with impaired renal clearance particularly for dabigatran and rivaroxaban. INR was in therapeutic range only $64 \%$ of the time in RELY, $62 \%$ of the time in ARISTOTLE, and $55 \%$ of the time in ROCKET-AF. While subanalyses of the RELY and ROCKET-AF studies showed benefit of the new agents compared to patients spending more time with INR in therapeutic range, these were post hoc analyses, raising some uncertainty about the benefit of these agents in patients who are already well controlled on warfarin $[15,16]$.

While effective from the point of view of preventing strokes and other embolic events, one must be aware of significant risk of bleeding associated with these agents used alone and, especially, in combination. Unfortunately neither has a specific reversal agent. It appears possible to remove dabigatran using hemodialysis and to reverse the other two agents with prothrombin complex concentrate which has no effect on the anticoagulant effect of dabigatran [17]. All agents showed a significant decrease in the dreaded intracranial hemorrhage compared to warfarin, but there was a trend to increased gastrointestinal bleeding with rivaroxaban and with the higher dose of dabigatran at $150 \mathrm{mg}$ twice per day.
One other strategy aiming to minimize the risk of embolic events involves mechanical elimination or closure of the left atrial appendage (LAA), the area where clots related to atrial fibrillation most commonly form. Techniques for LAA closure or excision have been initially developed by the cardiac surgeons [18]. Novel LAA closure devices have recently shown promise in reducing the risk of stroke in patients who cannot take antithrombotic agents and can be placed percutaneously [19]. In a large randomized study, these devices were shown to be non-inferior to warfarin with slight excess of periprocedural complications in the invasive arm of the study [20].

With "crowding" in the area of embolic prevention, selection of the best approach for the patient is becoming more problematic [19]. Several scores have been developed to predict both the risk of stroke and that of significant bleeding [21-23] yet the guidelines incorporating those scores provide little guidance among the low-to-intermediate risk patients with guideline "creep" towards recommending OAC for a greater stratum of patients, despite little new data on the risk of stroke, lower stroke risk for the same risk score in some recently reported studies [24], and evidence that combination of aspirin and clopidogrel is equivalent in stroke prevention to warfarin, provided patients spend $65 \%$ of the time or less in therapeutic range [25], a figure higher than that for the warfarin-treated arm of all studies showing equivalent or greater efficacy of the novel antithrombotic agents. Nevertheless, several professional organizations charged with creating practice guidelines rapidly adopted the novel agents as a standard of care, in some cases before the agents were actually approved for market use [26, 27].

Guidance to avoid anticoagulation in patients at high risk of bleeding makes therapeutic selection even more cumbersome with many of the risk factors listed as associated with increased bleeding rate also present in the risk scores used to estimate the hazard of thromboembolic events $[23,28]$.

Nevertheless, availability of the new oral anticoagulation agents with faster onset of action and better-defined kinetics as well as left atrial appendageal occlude devices will help standardize care of AF patients and make it less arduous. These innovations will help clinicians get away from the perils of bridging anticoagulation around invasive procedures in high-risk patients and prevent or shorten hospital admissions. Time and further studies will help better define the best of the new agents and devices and, perhaps, tailor therapy to individual patient specifics.

\section{Rate, Rhythm, or Quality of Life?}

From the outset of clinical investigation into AF management it made common sense to pursue normal sinus rhythm as the goal for most patients. It seemed only natural that patients in sinus rhythm should fare better than those in AF. A number of studies set out to compare outcomes in patients treated with the goal to achieve sinus rhythm or remain in atrial fibrillation with a controlled ventricular response. As a surprise to many, these studies uniformly showed little advantage to the strategy of rhythm maintenance. 
In Pharmacological Intervention in AF (PIAF) study, 252 patients with persistent AF were randomly assigned to diltiazem for rate control or amiodarone for maintenance of sinus rhythm [29]. All patients were anticoagulated to prevent embolic sequelae of the arrhythmia. There was no difference in the Quality of Life (QOL) measures at 1 year with some functional capacity improvement in patients treated with amiodarone at a cost of more hospital admissions in this group.

Strategies of Treatment of AF (STAF) randomized 200 patients with chronic AF, large left atria, heart failure symptoms, and mild-to-moderate LV dysfunction to amiodarone or a Class I antiarrhythmic drug versus rate control [30]. All patients were anticoagulated. While there was no difference in the likelihood of the composite endpoint of death or embolic event, there was a trend to higher risk of stroke in the rhythm control arm.

Rate Control versus Electrical Cardioversion (RACE) study randomized 522 patients with persistent AF to antiarrhythmic therapy with Sotalol, Class I medications or amiodarone versus rate control [31]. Patients in the rhythm control arm were anticoagulated only during the pericardioversion period. Clinical outcomes between the two strategies were similar at the conclusion of the trial.

The largest of the rate versus rhythm control studies, Atrial Fibrillation Follow-up Investigation of Rhythm Management (AFFIRM) sought to compare these strategies in 4060 patients with recurrent AF [32]. Anticoagulation could be stopped in the rhythm control arm if sinus rhythm were present for at least 4 weeks. Intention-to-treat analysis of the AFFIRM study demonstrated a trend to a lower combined endpoint of mortality, embolism, and hospitalization in the rate control arm of the trial.

Patients with congestive heart failure (CHF) would have been expected to derive the greatest benefit from sinus rhythm. These patients may be particularly sensitive to loss of atrial contraction and rapid ventricular rates, both interfering with left ventricular filling, already impaired in CHF. Nevertheless, in the Rhythm Control versus Rate Control for Atrial Fibrillation and Heart Failure (AF-CHF) study patients treated with amiodarone to achieve sinus rhythm fared no better than those treated with beta blockers and digoxin to achieve adequate rate control [33].

Findings of these studies are easier to interpret in the light of the limited efficacy of the rate and particularly rhythm control regimens. Only $38 \%$ of the patients in the rhythm control arm in STAF and 39\% of the patients assigned to rhythm control in RACE were in sinus rhythm. Patients in PIAF and AFFIRM had a slightly better response to therapy, but still, only two-thirds of the patients treated with amiodarone in PIAF were in sinus rhythm, on par with $63 \%$ percent of the patients assigned to rhythm control in AFFIRM and $73 \%$ of these patients in AF-CHF who were in sinus rhythm at the conclusion of the study. By comparison, two thirds of the rate control patients in PIAF and $80 \%$ of the rate control patients in AFFIRM and AF-CHF achieved prespecified ventricular response rates.

While the intention-to-treat analysis in RACE showed no difference in the QOL outcomes between patients assigned to rate or rhythm control, patients in sinus rhythm had better QOL in this study[34]. This was supported by the Canadian Trial of Atrial Fibrillation [35] where summary measures of physical and mental health improved significantly with treatment from baseline, regardless of therapy selection, with global well-being rated significantly lower by patients with recurrent AF compared to those without. Rhythm control strategy leads to better exercise tolerance and functional capacity in several trials, including the AFFIRM in-rhythm analysis [36]. Furthermore, when STAF investigators compared baseline quality of life measures in their subjects with those in background German population in sinus rhythm they found all SF-36 measures to be significantly lower among the study subjects.

Patients who were actually able to achieve sinus rhythm in AFFIRM also had a $47 \%$ mortality risk reduction compared to those who were in $\mathrm{AF}(P<0.0001)$ [37]. These findings were supported by meta-analyses of rate versus rhythm control trials $[38,39]$.

Why then did the patients who were treated with antiarrhythmic agents perform so poorly even though sinus rhythm itself was associated with superior outcomes? Part of the answer comes from the limited efficacy of these agents to actually control rhythm as described. The second part has to do with a significant adverse event profile of these medications and in particular amiodarone. All of these medications have been shown to increase mortality in certain populations, in particular among patients with ischemic heart disease and myocardial dysfunction, in the case of Sotalol [40] and Class IC agents [41], and among patients with left ventricular dysfunction and history of congestive heart failure, in the case of dronedarone [42, 43], a newcomer to this class of drugs. Amiodarone, while the most effective antiarrhythmic of all, has been shown to cause significant bradycardia in $5 \%$ of the patients, thyroid toxicity in $23 \%$, skin toxicity in up to $75 \%$, neurologic toxicity in up to $30 \%$, and corneal deposits in $100 \%$ of the treated patients [44]. Uniformly, close to $30 \%$ of the patients treated with amiodarone in these studies stopped the drug due to side effects.

Dronedarone, a Class III antiarrhythmic agent, was engineered based on the amiodarone molecule in hope of providing equivalent clinical benefit without multiple side effects [45]. Pharmacologically, the molecule of dronedarone does not carry iodine, thought to account for most of the end-organ toxicity seen among amiodarone patients. It was also modified to make it more hydrophilic and to expedite elimination half-life compared to its parent drug. Dronedarone had undergone extensive clinical testing in multiple trials and was shown to have rhythm control efficacy comparable to that of Sotalol or Class I C agents. In addition dronedarone has been shown to provide a measure of rate control, lowering heart rate in atrial fibrillation among treated patients by an average of 14 beats per minute [46]. Unlike other antiarrhythmic agents, dronedarone could be started on an outpatient basis without the need for inpatient monitoring required for Sotalol, Propafenone, Flecainide, and Dofetilide. ATHENA, a double-blind placebocontrolled trial, studied the effects of dronedarone in addition to standard therapy in patients with risk factors 
including age over 75 , or age under 75 with at least one of hypertension, diabetes, stroke or TIA, enlarged left atrial dimension $(>50 \mathrm{~mm})$, or reduced left ventricular ejection fraction $(<40 \%)$ [47]. Dronedarone was shown to reduce AF-related hospitalizations with a hazard ratio of 0.626 compared to placebo [48]. It also significantly reduced duration of hospitalization in these patients. These effects would potentially reduce the cost of care by 2875 Euro per patient per year based on the European health economics data or approximately $\$ 3000-6000$ based on similar US and Canadian data.

Dronedarone was quickly elevated to the status of firstline agent by some professional organizations [49], but soon proved neither nearly as effective as amiodarone [50, 51], nor as safe as it was touted to be $[52,53]$. In a recently published Permanent Atrial Fibrillation Outcome Study Using Dronedarone on Top of Standard Therapy (PALLAS), when administered to patients with high-risk permanent atrial fibrillation dronedarone was associated with doubling of mortality among treated patients, more than double the risk of stroke and nearly double the risk of heart failure hospitalization [43]. Unfortunately, guideline production cycle has not caught up to the recent developments, leaving clinicians in a quandary.

Several new agents are undergoing studies to determine their place in the antiarrhythmic line-up. Two of these, vernakalant and ranolazine, have substantial data behind them. Vernakalant predominantly blocks atrial potassium currents. Intravenous form of this medication has been shown to be effective in restoring sinus rhythm without significant proarrhythmic effect [54-56]. Ranolazine is a multichannel blocker approved for treatment of chronic angina. It has been shown to reduce the burden of atrial fibrillation and to have a synergistic effect with amiodarone and dronedarone [57-59].

Overall, medical therapy, whether aimed at control of the ventricular rate or rhythm, fails miserably and is poorly tolerated, in other words, medical rhythm control and rate control strategies are equally poor, rather than equally effective. Patients in AFFIRM and other medical rate versus rhythm control studies were not necessarily symptomaticthese latter patients were more likely excluded and offered rhythm control therapy. This enrolment bias further limits applicability of the findings of these studies to the clinical care of patients frequenting emergency rooms because of disabling symptoms stemming from their AF. Accordingly, selection of therapy in these patients should really focus on symptom improvement and quality of life rather than rate or rhythm control per se.

\section{Is the Strategy of Ablate and Pace a Good Answer to Medical Therapy for All Patients?}

Advent of ablation and cardiac rhythm devices signaled renewed hope for the victims of $\mathrm{AF}$ and added to the armamentarium of the treating clinician. These therapies could be applied for both rate and rhythm control indications. In the first instance, AV nodal ablation and permanent pacing significantly reduced cardiac symptoms, while improving exercise duration, quality of life, and ejection fraction, in a meta-analysis of clinical studies based on over 1000 patients [60]. In particular patients with chronic AF and congestive heart failure symptoms treated with AV nodal ablation derive a greater benefit from biventricular pacing with statistically significant improvement in functional capacity, when compared with RV pacing, as measured by the 6-minute walking test, peak VO2, and exercise duration [61].

Some of the explanation for the greater benefit of $\mathrm{AV}$ junction ablation approach come from the Rate Control Efficacy in Permanent AF (RACE II) trial of aggressive versus lenient rate control strategies. Aggressive strategy in this study targeted conventional rate control criteria used in AFFIRM of resting heart rate less than $80 \mathrm{bpm}$ and peak heart rate with moderate exercise of less than $110 \mathrm{bpm}$, while the lenient strategy targeted resting heart rate less than $110 \mathrm{bpm}$. In this study, patients assigned to strict heart rate control did substantially worse with a $16 \%$ greater risk of the composite of cardiovascular death, heart failure hospitalization, stroke, systemic embolism, bleeding, or "lifethreatening arrhythmic events" defined as syncope, sustained VT, cardiac arrest, life-threatening adverse events of ratecontrol drugs, or pacemaker or ICD implantation, driven by greater rates of bradycardia-related complications. Moreover $45 \%$ of the patients in both groups still had significant AFrelated symptoms at the end of the study [62].

Ablate and pace approach is very effective among the older patients with tachycardia-bradycardia syndrome who are intolerant or have contraindications to a number of medications unfortunately, this strategy is frequently applied to younger patients with greater burden of device-related complications over the lifetime. Patients thus treated may require device replacement and upgrade down the road, some may develop RV-pacing-mediated cardiomyopathy, yet others might suffer a worse fate, succumbing to sudden cardiac death as a result of pacing system failure or overly rapid down titration of the ventricular rate and ensuing ventricular arrhythmia [63]. Unlike selection of medical therapy for patients with symptomatic AF, quality of life notwithstanding, downstream considerations should guide selection of this approach.

\section{To Burn or Not to Burn?}

Catheter ablation has rapidly moved to the mainstream of AF therapy over the past decade. This approach is based on the notion that paroxysmal AF episodes arise as a result of focal firing in the pulmonary veins and elsewhere in the left and right atria [64]. While initially considered "curative," over the last few years it is becoming apparent that many patients treated in this fashion return with further episodes of arrhythmia down the road [65], and many more continue to experience asymptomatic episodes of arrhythmia [66]. Nevertheless, studies of AF ablation have uniformly found improved quality of life among ablated patients.

A retrospective study in 1171 consecutive patients in their mid-sixties referred with symptomatic AF was one of the first 
to address QOL improvement with ablation compared to medical therapy [67]. Circumferential pulmonary vein ablation was performed in 589 patients with the remainder treated medically, aiming at rhythm control. At 900 days of followup, patients treated with ablation had a 70\% reduction in the likelihood of AF recurrence. Apart from an impressive improvement in survival among the ablated patients, the investigators reported similar changes in the QOL measures in both groups, with AF recurrences associated with significant reductions in physical and mental functioning in the medical group and impaired psychological well-being among the ablated patients. Similar improvements in survival and reduction in the risk of stroke among ablated patients were seen in a registry of patients treated in UK and Australia when compared to those treated medically in the Euro-Heart Survey [68].

A randomized trial of Radiofrequency Ablation versus Antiarrhythmic Drugs as First-line Treatment of Symptomatic Atrial Fibrillation (RAAFT) enrolled 70 patients in their mid-fifties with paroxysmal AF in 2001-2002 [69]. Within one year of followup, AF recurrence was five times less likely in patients randomized to ablation compared to those treated medically. Improvement in the QOL measures was more pronounced in the invasive arm of the study in five out of eight subclasses of the SF-36 scale.

While most patients undergoing AF ablation are young with little structural heart disease and largely paroxysmal AF, this therapy has been applied to patients with structural heart disease as well as those with persistent and chronic AF. In a study by Chen and colleagues, 94 patients with ejection fraction below $40 \%$ were ablated at the Cleveland Clinic between 2000 and 2003 [70]. After the first procedure, $73 \%$ of the patients with low ejection fraction were free of AF recurrence at $14 \pm 6$ months. Six months after the procedure, significant improvement in the areas of general health, energy, physical functioning, and emotional wellbeing was reported by the patients.

Hsu and colleagues studied 58 consecutive patients with ejection fraction less than $45 \%$ and clinical congestive heart failure symptoms [71]. After $12 \pm 7$ months, $78 \%$ of these patients remained in sinus rhythm with $21 \pm 13 \%$ improvement in ejection fraction, even though rate control prior to ablation was considered adequate in these patients. Restoration and maintenance of sinus rhythm along with cardiac structural improvement in these patients were paralleled by improvement in measures of QOL and functional performance with NYHA functional class improving on average by one grade 12 months following the procedure. Improvement was seen in the areas of physical and mental function, as well as exercise time and capacity.

Looking at this data it may appear that just about anyone may benefit from this approach. The truth is that AF ablation primarily targets pulmonary vein antra-a known culprit responsible for arrhythmia among patients with paroxysmal AF. It should not come as a surprise that patients with persistent and chronic AF while frequently ablated do not do as well as their paroxysmal counterparts, with about double the rate of long-term failure of the procedure [65].
A number of studies looked at additional ablation strategies divided into those that create linear lesions between the veins and extending from the veins to the mitral annulus. In a study by Earley and colleagues, 42 patients with permanent $\mathrm{AF}$ were treated with circumferential pulmonary vein ablation with isolation verified using noncontact mapping approach [72]. In addition to isolating the veins, the investigators delivered linear lesions across the left atrial roof and the cavotricuspid isthmus. These procedures were time consuming at close to 5 hours and required extensive fluoroscopy despite $3 \mathrm{D}$ mapping. $52 \%$ of the patients had to have a redo ablation with $74 \%$ success after multiple procedures. In a larger randomized study 142 patients received amiodarone and had a cardioversion with or without antral ablation [73]. The authors reported $74 \%$ success of the ablation approach at 12 months with $32 \%$ of the patients requiring repeat ablation. Only $3 \%$ of the patients assigned to amiodarone and a cardioversion alone maintaining sinus rhythm.

Another approach involves ablation of the areas demonstrating continuous fractionated electrical (CFE) activity during atrial fibrillation. While successful in the hands of some operators with $66 \%$ freedom from $\mathrm{AF}$ at one year off antiarrhythmic drugs (30\% of the patients requiring a second ablation to achieve this goal) [74], this approach is difficult to reproduce because of great interoperator variability in defining target electrograms as well lack of a consistent ablation endpoint across studies. So in a study of 66 patients with LA CFE ablation only $22 \%$ converted to sinus rhythm with this approach [75]. These patients experienced $70 \%$ long-term freedom from AF. On the contrary, patients remaining in $\mathrm{AF}$ after CFE ablation in the left atrium who were then randomly assigned to cardioversion or additional CFE ablation in the coronary sinus and the right atrium experienced a low success rate under 30\% in both groups. More recent work employing automated standardized software algorithms to identify the substrate have borne results reproducible across multiple centers and supported by at least two meta-analyses [76-78].

The other line or work aimed to identify neural mechanisms responsible for triggering and sustaining AF particularly in patients with a more persistent arrhythmia. Here, parasympathetic ganglia adjacent to the left atrium were shown in bench research to mediate AF [79]. While in an elegant animal study ablation of these ganglia was sufficient in terminating $\mathrm{AF}$ and rendering it noninducible [80], human data is limited primarily to an approach combining pulmonary vein antrum isolation with ablation targeting parasympathetic ganglionic plexi [81].

Some operators have included the right atrium and the coronary sinus in the lesion set. In one such study, ablation in the right atrium combined a posterior intercaval line with a septal line and SVC isolation. The results were startling, with over $80 \%$ success with the combined approach compared to $60 \%$ success with left atrial lesions alone [82].

Obviously, grouping of strategies into a prescribed "one size-fits-all" approach is too simplistic when applied to patients with chronic atrial fibrillation, likely stemming from a variety of mechanisms, not uniformly distributed across treated patients. A more intuitive approach, which has been 
gaining popularity, involves stepwise ablation of several targets with endpoint of AF conversion and arrhythmia termination [83]. First, pulmonary vein antra are isolated, then extensive defragmentation takes place on the roof, septum, and floor of the left atrium as well as at the base of the left atrial appendage. Finally, ablation of the mitral isthmus takes place with documentation of bidirectional block across all of the linear ablation sets. If the arrhythmia converts from $\mathrm{AF}$ into an atrial tachycardia or flutter, this is mapped and ablated. Ablation may also involve targets throughout the coronary sinus and in the right atrium, such as the SVC, the cavotricuspid isthmus, interatrial septum, and the crista terminalis. Using this approach AF has been shown to convert to either sinus rhythm or a more organized tachyarrhythmia in over $87 \%$ of the patients with $95 \%$ 1-year freedom from arrhythmia after 1.4 procedures/patient.

AF ablation is not without risk. While reported in each individual study, the likelihood of experiencing a complication has been best illustrated in the Worldwide Survey of Atrial Fibrillation Ablation [84]. The authors demonstrated total risk of serious complication to total $4.5 \%$ with $25(0.15 \%)$ deaths, $213(1.31 \%)$ tamponade, and $152(0.94 \%)$ strokes or TIAs in 20825 catheter ablation procedures performed in 16309 patients across 182 centers. Subject to recall bias, these findings come very close to those of a recently published study of AF ablation outcomes based on an insurance database from California, where $5 \%$ of the patients had periprocedural complications [85]. The authors reported 1 death $(0.02 \%), 13$ strokes or TIAs $(0.31 \%)$, and $104(2.5 \%)$ tamponades among 4156 patients undergoing ablation "in the real world". Unlike the negative publicity towards AF ablation that was evoked in the media by this paper, the findings are indeed positive, suggesting that AF ablation is as safe as we believed it to be despite relatively little individual center experience at a mean of 15.4 procedures per center per year in the study.

Most of the studies to date have reported outcomes of solid tip catheter ablation or ablation using a saline-irrigated tip catheter. A number of new technologies are rapidly becoming available to simplify AF ablation by allowing for energy delivery in a circumferential fashion around PV ostia. These include the PVAC catheter (Ablation Frontiers, Medtronic Inc., Minneapolis, Minnesota), utilizing dutycycled multipolar RF energy delivery, Mesh Ablator (Bard) using unipolar RF and cryoballoon ablation (Arctic Front, Medtronic Inc., Minneapolis, Minnesota). The principal driver for these developments is the hope that the new technologies will decrease procedure time and the need for fluoroscopy, maintain or improve procedural efficacy and safety, and make AF ablation more accessible with shorter learning curves.

Two recently published single-centre randomized studies compared AF ablation using PVAC with wide-area circumferential pulmonary vein ablation guided by Ensite Navx in one study and the CARTO system in the other [86, 87]. Both studies showed equivalent clinical outcomes with shorter procedure, radiofrequency, and fluoroscopy times achieved in patients treated with multipolar ablation. In a nonrandomised comparison of the two approaches currently in press, Tivig et al. [88] ablated 420 patients, 209 of them using PVAC. Duty-cycled RF was delivered on the septum and elsewhere in the left atrium using other multipolar ablation catheters in this group. Similarly, more extensive ablation was performed on patients treated historically at the investigators'center using 3D guided approach. This involved ablation of the roof and the mitral isthmus lines without seeking proof of block across these lines in all patients and CFE ablation among patients with persistent AF. The authors found both techniques equivalent with respect to long-term outcomes and procedural characteristics.

One must be cautious interpreting these results and consider recent evidence suggesting that ablation using dutycycled multipolar RF may lead to a greater cerebral ischemic burden compared to conventional irrigated ablation. In a study reported by Herrera Siklódy et al. [89], 37.5\% of the patients ablated using PVAC had new cerebroembolic lesions detected by acute postprocedural magnetic resonance imaging, compared to $7.4 \%$ of the patients ablated using irrigated point-by-point RF energy delivery and $4.3 \%$ of the patients undergoing balloon cryoablation; many of these lesions could still be seen months following ablation [90]. This was supported by the findings reported by Gaita et al. (38.9\% with PVAC versus $8.3 \%$ with irrigated RF and $5.6 \%$ with balloon cryoablation [91]). These concerns were recently rekindled in the context of the Tailored Treatment of Permanent Atrial Fibrillation (TTOP-AF) study presented at the 12th International Workshop on Cardiac Arrhythmias in Venice in October 2011, where 4 (2.9\%) of the 138 patients ablated using this technology had full-blown clinical strokes, a much greater risk than that reported in other studies.

All in all, although not perfect, ablation of AF appears to be effective in terms of improving quality of life, particularly among patients with paroxysmal atrial fibrillation, with an acceptable safety profile. New ablation protocols and technologies will hopefully lead to improved outcomes over time, but should be adopted cautiously and after a thorough evaluation in rigorous clinical trials. More intense monitoring following the procedure may help us understand the true burden of recurrent $\mathrm{AF}$ following ablation and routine application of validated QOL scales to evaluate patients at every step of the way will better describe the degree of symptomatic improvement experienced by the patients. Studies into whether or not ablation results in improved morbidity or mortality among treated patients are currently on the way.

\section{Conclusions}

Atrial fibrillation is a common condition with numerous clinical implications. Medical and invasive strategies in $\mathrm{AF}$ care are evolving rapidly and may be difficult to follow for the front-line clinician. Further studies are on the way to address controversies surrounding some of these approaches. In this environment, while it is very important for the professional organizations to rapidly review and adjust clinical practice guidelines as new therapies become available. Mechanisms to rapidly distribute these guidelines to the clinicians must be firmly in place for the innovation to translate into better 
standards of care along with safeguards precluding premature adoption of therapies.

\section{Abbreviations}

AF: Atrial fibrillation

PV: Pulmonary vein

PVAI: Pulmonary vein antrum isolation

QOL: Quality of life

OAC: Oral anticoagulation

TIA: Transient ischemic attack

CHF: Congestive heart failure

CFE: Continuous (complex) fractionated electrograms

INR: International normalized ratio.

\section{References}

[1] T. Lewis, Clinical Disorders of the Heart Beat, Shaw \& Sons, 1925.

[2] D. Baily, M. H. Lehmann, D. N. Schumacher et al., "Hospitalization for arrhythmias in the United States: importance of atrial fibrillation," Journal of the American College of Cardiology, vol. 19, no. 3, supplement, article 41, 1992.

[3] P. A. Wolf, J. B. Mitchell, C. S. Baker, W. B. Kannel, and R. B. D'Agostino, "Impact of atrial fibrillation on mortality, stroke, and medical costs," Archives of Internal Medicine, vol. 158, no. 3, pp. 229-234, 1998.

[4] E. J. Benjamin, P. A. Wolf, R. B. D’Agostino, H. Silbershatz, W. B. Kannel, and D. Levy, "Impact of atrial fibrillation on the risk of death: the Framingham Heart Study," Circulation, vol. 98, no. 10, pp. 946-952, 1998.

[5] W. B. Kannel, P. A. Wolf, E. J. Benjamin, and D. Levy, "Prevalence, incidence, prognosis, and predisposing conditions for atrial fibrillation: population-based estimates," The American Journal of Cardiology, vol. 82, no. 7, supplement 1, pp. 2N-9N, 1998.

[6] A. S. Go, E. M. Hylek, K. A. Phillips et al., "Prevalence of diagnosed atrial fibrillation in adults: national implications for rhythm management and stroke prevention: the anticoagulation and risk factors in atrial fibrillation ((ATRIA) study," Journal of the American Medical Association, vol. 285, no. 18, pp. 2370-2375, 2001.

[7] S. Stewart, N. F. Murphy, A. Walker, A. McGuire, and J. J. V. McMurray, "Cost of an emerging epidemic: an economic analysis of atrial fibrillation in the UK," Heart, vol. 90, pp. 286292, 2004.

[8] T. Reinhold, C Lindig, S. N. Willich, and B. Bruggenjurgen, "The costs of atrial fibrillation in patients with cardiovascular comorbidities - a longitudinal analysis of German health insurance data," Europace, vol. 13, no. 9, pp. 1275-1280, 2011.

[9] R. G. Hart, L. A. Pearce, and M. I. Aguilar, "Meta-analysis: antithrombotic therapy to prevent stroke in patients who have nonvalvular atrial fibrillation," Annals of Internal Medicine, vol. 146, no. 12, pp. 857-867, 2007.

[10] S. J. Connolly, J. Pogue, R. G. Hart et al., "Effect of clopidogrel added to aspirin in patients with atrial fibrillation," The New England Journal of Medicine, vol. 360, no. 20, pp. 2066-2078, 2009.

[11] S. J. Connolly, M. D. Ezekowitz, S. Yusuf et al., "Dabigatran versus warfarin in patients with atrial fibrillation," The New
England Journal of Medicine, vol. 361, no. 12, pp. 1139-1151, 2009.

[12] D. J. Gladstone, E. Bui, J. Fang et al., "Potentially preventable strokes in high-risk patients with atrial fibrillation who are not adequately anticoagulated," Stroke, vol. 40, no. 1, pp. 235-240, 2009.

[13] C. B. Granger, J. H. Alexander, J. J. V. McMurray et al., "Apixaban versus warfarin in patients with atrial fibrillation," The New England Journal of Medicine, vol. 365, no. 11, pp. 981992, 2011.

[14] M. R. Patel, K. W. Mahaffey, J. Garg et al., "Rivaroxaban versus warfarin in nonvalvular atrial fibrillation," The New England Journal of Medicine, vol. 365, no. 10, pp. 883-891, 2011.

[15] L. Wallentin, S. Yusuf, M. D. Ezekowitz et al., "Efficacy and safety of dabigatran compared with warfarin at different levels of international normalised ratio control for stroke prevention in atrial fibrillation: an analysis of the RE-LY trial," The Lancet, vol. 376, no. 9745, pp. 975-983, 2010.

[16] Johnson \& Johnson Pharmaceutical Research \& Development, Advisory Committee Briefing Document, 2012.

[17] E. S. Eerenberg, P. W. Kamphuisen, M. K. Sijpkens, J. C. Meijers, H. R. Buller, and M. Levi, "Reversal of rivaroxaban and dabigatran by prothrombin complex concentrate: a randomized, placebo-controlled, crossover study in healthy subjects," Circulation, vol. 124, no. 14, pp. 1573-1579, 2011.

[18] J. L. Blackshear and J. A. Odell, "Appendage obliteration to reduce stroke in cardiac surgical patients with atrial fibrillation," The Annals of Thoracic Surgery, vol. 61, no. 2, pp. 755759, 1996.

[19] V. Y. Reddy, D. Holmes, S. K. Doshi, P. Neuzil, and S. Kar, "Safety of percutaneous left atrial appendage closure: results from the watchman left atrial appendage system for embolic protection in patients with AF (PROTECT AF) clinical trial and the continued access registry," Circulation, vol. 123, no. 4, pp. 417-424, 2011.

[20] D. R. Holmes, V. Y. Reddy, Z. G. Turi et al., "Percutaneous closure of the left atrial appendage versus warfarin therapy for prevention of stroke in patients with atrial fibrillation: a randomised non-inferiority trial," The Lancet, vol. 374, no. 9689, pp. 534-542, 2009.

[21] B. F. Gage, A. D. Waterman, W. Shannon, M. Boechler, M. W. Rich, and M. J. Radford, "Validation of clinical classification schemes for predicting stroke: results from the national registry of atrial fibrillation," The Journal of the American Medical Association, vol. 285, no. 22, pp. 2864-2870, 2001.

[22] G. Y. H. Lip, L. Frison, J. L. Halperin, and D. A. Lane, "Identifying patients at high risk for stroke despite anticoagulation: a comparison of contemporary stroke risk stratification schemes in an anticoagulated atrial fibrillation cohort," Stroke, vol. 41, no. 12, pp. 2731-2738, 2010.

[23] R. Pisters, D. A. Lane, R. Nieuwlaat, C. B. de Vos, H. J. G. M. Crijns, and G. Y. H. Lip, "A novel user-friendly score (HASBLED) to assess 1-year risk of major bleeding in patients with atrial fibrillation: the euro heart survey," Chest, vol. 138, no. 5, pp. 1093-1100, 2010.

[24] J. S. Healey, S. J. Connolly, M. R. Gold et al., "Subclinical atrial fibrillation and the risk of stroke," The New England Journal of Medicine, vol. 366, no. 2, pp. 120-129, 2012.

[25] S. J. Connolly, J. Pogue, J. Eikelboom et al., "Benefit of oral anticoagulant over antiplatelet therapy in atrial fibrillation depends on the quality of international normalized ratio control achieved by centers and countries as measured by time in therapeutic range," Circulation, vol. 118, no. 20, pp. 20292037, 2008. 
[26] J. A. Cairns, S. Connolly, S. McMurtry, M. Stephenson, and M. Talajic, "Canadian cardiovascular society atrial fibrillation guidelines 2010: prevention of stroke and systemic thromboembolism in atrial fibrillation and flutter," Canadian Journal of Cardiology, vol. 27, no. 1, pp. 74-90, 2011.

[27] A. J. Camm, P. Kirchhof, G. Y. H. Lip et al., "Guidelines for the management of atrial fibrillation: the task force for the management of atrial fibrillation of the European society of cardiology (ESC)," European Heart Journal, vol. 31, no. 19, pp. 2369-2429, 2010.

[28] M. C. Fang, A. S. Go, Y. Chang et al., "A new risk scheme to predict warfarin-associated hemorrhage: the ATRIA (anticoagulation and risk factors in atrial fibrillation) study," Journal of the American College of Cardiology, vol. 58, no. 10, pp. 395401, 2011.

[29] S. H. Hohnloser, K. H. Kuck, and J. Lilienthal, "Rhythm or rate control in atrial fibrillation-pharmacological intervention in atrial fibrillation (PIAF): a randomised trial," The Lancet, vol. 356, no. 9244, pp. 1789-1794, 2000.

[30] J. Carlsson, S. Miketic, J. Windeler et al., "Randomized trial of rate-control versus rhythm-control in persistent atrial fibrillation: the strategies of treatment of atrial fibrillation (STAF) study," Journal of the American College of Cardiology, vol. 41, no. 10, pp. 1690-1696, 2003.

[31] I. C. Van Gelder, V. E. Hagens, H. A. Bosker et al., "A comparison of rate control and rhythm control in patients with recurrent persistent atrial fibrillation," The New England Journal of Medicine, vol. 347, no. 23, pp. 1834-1840, 2002.

[32] D. G. Wyse, A. L. Waldo, J. P. DiMarco et al., "A comparison of rate control and rhythm control in patients with atrial fibrillation," The New England Journal of Medicine, vol. 347, no. 23, pp. 1825-1833, 2002.

[33] D. Roy, M. Talajic, S. Nattel et al., "Rhythm control versus rate control for atrial fibrillation and heart failure," The New England Journal of Medicine, vol. 358, no. 25, pp. 2667-2677, 2008.

[34] V. E. Hagens, A. V. Ranchor, E. Van Sonderen et al., "Effect of rate or rhythm control on quality of life in persistent atrial fibrillation: results from the rate control versus electrical cardioversion (RACE) study," Journal of the American College of Cardiology, vol. 43, no. 2, pp. 241-247, 2004.

[35] P. Dorian, M. Paquette, D. Newman et al., "Quality of life improves with treatment in the Canadian trial of atrial fibrillation," American Heart Journal, vol. 143, no. 6, pp. 984990, 2002.

[36] M. K. Chung, L. Shemanski, D. G. Sherman et al., "Functional status in rate- versus rhythm-control strategies for atrial fibrillation: results of the atrial fibrillation follow-up investigation of rhythm management (AFFIRM) functional status substudy," Journal of the American College of Cardiology, vol. 46, no. 10, pp. 1891-1899, 2005.

[37] S. D. Corley, A. E. Epstein, J. P. DiMarco et al., "Relationships between sinus rhythm, treatment, and survival in the atrial fibrillation follow-up investigation of rhythm management (AFFIRM) study," Circulation, vol. 109, no. 12, pp. 1509-1513, 2004.

[38] L. Testa, G. G. L. Biondi-Zoccai, A. D. Russo, F. Bellocci, F. Andreotti, and F. Crea, "Rate-control vs. rhythm-control in patients with atrial fibrillation: a meta-analysis," European Heart Journal, vol. 26, no. 19, pp. 2000-2006, 2005.

[39] S. de Denus, C. A. Sanoski, J. Carlsson, G. Opolski, and S. A. Spinler, "Rate vs rhythm control in patients with atrial fibrillation: a meta-analysis," Archives of Internal Medicine, vol. 165 , no. 3, pp. 258-262, 2005.
[40] A. L. Waldo, A. J. Camm, H. deRuyter et al., "Effect of d-sotalol on mortality in patients with left ventricular dysfunction after recent and remote myocardial infarction. The SWORD investigators. Survival with oral d-sotalol," The Lancet, vol. 348, no. 9019, pp. 7-12, 1996.

[41] The Cardiac Arrhythmia Suppression Trial (CAST) Investigators, "Preliminary report: effect of encainide and flecainide on mortality in a randomized trial of arrhythmia suppression after myocardial infarction," The New England Journal of Medicine, vol. 321, no. 6, pp. 406-412, 1989.

[42] L. Køber, C. Torp-Pedersen, J. J. V. McMurray et al., "Increased mortality after dronedarone therapy for severe heart failure," The New England Journal of Medicine, vol. 358, no. 25, pp. 2678-2687, 2008.

[43] S. J. Connolly, A. J. Camm, J. L. Halperin et al., "Dronedarone in high-risk permanent atrial fibrillation," The New England Journal of Medicine, vol. 365, no. 24, pp. 2268-2276, 2011.

[44] P. Zimetbaum, "Amiodarone for atrial fibrillation," The New England Journal of Medicine, vol. 356, no. 9, pp. 935-941, 2007.

[45] S. Kathofer, D. Thomas, and C. A. Karle, "The novel antiarrhythmic drug dronedarone: comparison with amiodarone," Cardiovascular Drug Reviews, vol. 23, no. 3, pp. 217-230, 2005.

[46] J. M. Davy, M. Herold, C. Hoglund et al., "Dronedarone for the control of ventricular rate in permanent atrial fibrillation: the efficacy and safety of dRonedArone for The cOntrol of ventricular rate during atrial fibrillation (ERATO) study," American Heart Journal, vol. 156, no. 3, pp. 527.e1-527.e9, 2008.

[47] S. H. Hohnloser, H. J. G. M. Crijns, M. van Eickels et al., "Effect of dronedarone on cardiovascular events in atrial fibrillation," The New England Journal of Medicine, vol. 360, no. 7, pp. 668-678, 2009.

[48] C. Torp-Pedersen, H. J. Crijns, C. Gaudin, R. L. Page, S. J. Connolly, and S. H. Hohnloser, "Impact of dronedarone on hospitalization burden in patients with atrial fibrillation: results from the ATHENA study," Europace, vol. 13, no. 8, pp. 1118-1126, 2011.

[49] A. M. Gillis, A. Verma, M. Talajic, S. Nattel, and P. Dorian, "Canadian cardiovascular society atrial fibrillation guidelines 2010: rate and rhythm management," Canadian Journal of Cardiology, vol. 27, no. 1, pp. 47-59, 2011.

[50] J. P. Piccini, V. Hasselblad, E. D. Peterson, J. B. Washam, R. M. Califf, and D. F. Kong, "Comparative efficacy of dronedarone and amiodarone for the maintenance of sinus rhythm in patients with atrial fibrillation," Journal of the American College of Cardiology, vol. 54, no. 12, pp. 1089-1095, 2009.

[51] J. Y. Le Heuzey, G. M. De Ferrari, D. Radzik, M. Santini, J. Zhu, and J. M. Davy, "A short-term, randomized, doubleblind, parallel-group study to evaluate the efficacy and safety of dronedarone versus amiodarone in patients with persistent atrial fibrillation: the dionysos study," Journal of Cardiovascular Electrophysiology, vol. 21, no. 6, pp. 597-605, 2010.

[52] FDA, "FDA Drug Safety Communication: Multaq (dronedarone) and increased risk of death and serious cardiovascular adverse events," 2011.

[53] FDA, "FDA Drug Safety Communication: severe liver injury associated with the use of dronedarone (marketed as Multaq)," 2011.

[54] D. Roy, C. M. Pratt, C. Torp-Pedersen et al., "Vernakalant hydrochloride for rapid conversion of atrial fibrillation: a phase 3, randomized, placebo-controlled trial," Circulation, vol. 117, no. 12, pp. 1518-1525, 2008. 
[55] P. R. Kowey, P. Dorian, L. B. Mitchell et al., "Vernakalant hydrochloride for the rapid conversion of atrial fibrillation after cardiac surgery a randomized, double-blind, placebocontrolled trial," Circulation, vol. 2, no. 6, pp. 652-659, 2009.

[56] A. J. Camm, A. Capucci, S. H. Hohnloser et al., "A randomized active-controlled study comparing the efficacy and safety of vernakalant to amiodarone in recent-onset atrial fibrillation," Journal of the American College of Cardiology, vol. 57, no. 3, pp. 313-321, 2011.

[57] B. M. Scirica, D. A. Morrow, H. Hod et al., "Effect of ranolazine, an antianginal agent with novel electrophysiological properties, on the incidence of arrhythmias in patients with non-ST-segment-elevation acute coronary syndrome: results from the metabolic efficiency with ranolazine for less ischemia in non-ST-elevation acute coronary syndrome-thrombolysis in myocardial infarction 36 (MERLIN-TIMI 36) randomized controlled trial," Circulation, vol. 116, no. 15, pp. 1647-1652, 2007.

[58] A. Burashnikov, S. Sicouri, J. M. Di Diego, L. Belardinelli, and C. Antzelevitch, "Synergistic effect of the combination of ranolazine and dronedarone to suppress atrial fibrillation," Journal of the American College of Cardiology, vol. 56, no. 15, pp. 1216-1224, 2010.

[59] S. Sicouri, A. Burashnikov, L. Belardinelli, and C. Antzelevitch, "Synergistic electrophysiologic and antiarrhythmic effects of the combination of ranolazine and chronic amiodarone in canine atria," Circulation, vol. 3, no. 1, pp. 88-95, 2010.

[60] M. A. Wood, C. Brown-Mahoney, G. N. Kay, and K. A. Ellenbogen, "Clinical outcomes after ablation and pacing therapy for atrial fibrillation: a meta-analysis," Circulation, vol. 101, no. 10, pp. 1138-1144, 2000.

[61] R. N. Doshi, E. G. Daoud, C. Fellows et al., "Left ventricularbased cardiac stimulation post AV nodal ablation evaluation (The PAVE study)," Journal of Cardiovascular Electrophysiology, vol. 16, no. 11, pp. 1160-1165, 2005.

[62] I. C. Van Gelder, H. F. Groenveld, H. J. G. M. Crijns et al., "Lenient versus strict rate control in patients with atrial fibrillation," The New England Journal of Medicine, vol. 362, no. 15, pp. 1363-1373, 2010.

[63] C. Ozcan, A. Jahangir, P. A. Friedman et al., "Sudden death after radiofrequency ablation of the atrioventricular node in patients with atrial fibrillation," Journal of the American College of Cardiology, vol. 40, no. 1, pp. 105-110, 2002.

[64] M. Haïssaguerre, P. Jaïs, D. C. Shah et al., "Spontaneous initiation of atrial fibrillation by ectopic beats originating in the pulmonary veins," The New England Journal of Medicine, vol. 339, no. 10, pp. 659-666, 1998.

[65] Y. Khaykin, R. Oosthuizen, L. Zarnett et al., "Clinical predictors of arrhythmia recurrences following pulmonary vein antrum isolation for atrial fibrillation: predicting arrhythmia recurrence post-PVAI," Journal of Cardiovascular Electrophysiology, vol. 22, no. 11, pp. 1206-1214, 2011.

[66] A. Verma, J. Champagne, J. Sapp et al., "Discerning the incidence of symptomatic and asymptomatic episodes of atrial fibrillation pre- and post- radiofrequency ablation (discern af): a prospective, multicenter study," in Proceedings of the 32nd Annual Scientific Sessions of the Heart Rhythm Society, 2011.

[67] C. Pappone, S. Rosanio, G. Augello et al., "Mortality, morbidity, and quality of life after circumferential pulmonary vein ablation for atrial fibrillation: outcomes from a controlled nonrandomized long-term study," Journal of the American College of Cardiology, vol. 42, no. 2, pp. 185-197, 2003.
[68] R. J. Hunter, J. McCready, I. Diab et al., "Maintenance of sinus rhythm with an ablation strategy in patients with atrial fibrillation is associated with a lower risk of stroke and death," Heart, vol. 98, no. 1, pp. 48-53, 2012.

[69] O. M. Wazni, N. F. Marrouche, D. O. Martin et al., "Radiofrequency ablation vs antiarrhythmic drugs as first-line treatment of symptomatic atrial fibrillation: a randomized trial," Journal of the American Medical Association, vol. 293, no. 21, pp. 2634-2640, 2005.

[70] M. S. Chen, N. F. Marrouche, Y. Khaykin et al., "Pulmonary vein isolation for the treatment of atrial fibrillation in patients with impaired systolic function," Journal of the American College of Cardiology, vol. 43, no. 6, pp. 1004-1009, 2004.

[71] L. F. Hsu, P. Jaïs, P. Sanders et al., "Catheter ablation for atrial fibrillation in congestive heart failure," The New England Journal of Medicine, vol. 351, no. 23, pp. 2373-2383, 2004.

[72] M. J. Earley, D. J. R. Abrams, A. D. Staniforth, S. C. Sporton, and R. J. Schilling, "Catheter ablation of permanent atrial fibrillation: medium term results," Heart, vol. 92, no. 2, pp. 233238, 2006.

[73] H. Oral, C. Pappone, A. Chugh et al., "Circumferential pulmonary-vein ablation for chronic atrial fibrillation," The New England Journal of Medicine, vol. 354, no. 9, pp. 934-941, 2006.

[74] K. Nademanee, J. McKenzie, E. Kosar et al., "A new approach for catheter ablation of atrial fibrillation: mapping of the electrophysiologic substrate," Journal of the American College of Cardiology, vol. 43, no. 11, pp. 2044-2053, 2004.

[75] H. Oral, A. Chugh, E. Good et al., "Randomized evaluation of right atrial ablation after left atrial ablation of complex fractionated atrial electrograms for long-lasting persistent atrial fibrillation," Circulation, vol. 1, no. 1, pp. 6-13, 2008.

[76] A. Verma, R. Mantovan, L. Macle et al., "Substrate and trigger ablation for reduction of atrial fibrillation (STAR AF): a randomized, multicentre, international trial," European Heart Journal, vol. 31, no. 11, pp. 1344-1356, 2010.

[77] M. H. Kong, J. P. Piccini, and T. D. Bahnson, "Efficacy of adjunctive ablation of complex fractionated atrial electrograms and pulmonary vein isolation for the treatment of atrial fibrillation: a meta-analysis of randomized controlled trials," Europace, vol. 13, no. 2, pp. 193-204, 2011.

[78] R. M. Hayward, G. A. Upadhyay, T. Mela et al., "Pulmonary vein isolation with complex fractionated atrial electrogram ablation for paroxysmal and nonparoxysmal atrial fibrillation: a meta-analysis," Heart Rhythm, vol. 8, no. 7, pp. 994-1000, 2011.

[79] J. Zhou, B. J. Scherlag, J. Edwards, W. M. Jackman, R. Lazzara, and S. S. Po, "Gradients of atrial refractoriness and inducibility of atrial fibrillation due to stimulation of ganglionated plexi," Journal of Cardiovascular Electrophysiology, vol. 18, no. 1, pp. 83-90, 2007.

[80] K. Lemola, D. Chartier, Y. H. Yeh et al., "Pulmonary vein region ablation in experimental vagal atrial fibrillation : role of pulmonary veins versus autonomic ganglia," Circulation, vol. 117, no. 4, pp. 470-477, 2008.

[81] H. Nakagawa, B. J. Scherlag, E. Patterson, A. Ikeda, D. Lockwood, and W. M. Jackman, "Pathophysiologic basis of autonomic ganglionated plexus ablation in patients with atrial fibrillation," Heart Rhythm, vol. 6, no. 12, pp. S26-S34, 2009.

[82] L. Calò, F. Lamberti, M. L. Loricchio et al., "Left atrial ablation versus biatrial ablation for persistent and permanent atrial fibrillation. A prospective and randomized study," Journal of the American College of Cardiology, vol. 47, no. 12, pp. 25042512, 2006. 
[83] M. Haïssaguerre, P. Sanders, M. Hocini et al., "Catheter ablation of long-lasting persistent atrial fibrillation: critical structures for termination," Journal of Cardiovascular Electrophysiology, vol. 16, no. 11, pp. 1125-1137, 2005.

[84] R. Cappato, H. Calkins, S. A. Chen et al., "Updated worldwide survey on the methods, efficacy, and safety of catheter ablation for human atrial fibrillation," Circulation, vol. 3, no. 1, pp. 3238, 2010.

[85] R. U. Shah, J. V. Freeman, D. Shilane, P. J. Wang, A. S. Go, and M. A. Hlatky, "Procedural complications, rehospitalizations, and repeat procedures after catheter ablation for atrial fibrillation," Journal of the American College of Cardiology, vol. 59, no. 2, pp. 143-149, 2012.

[86] A. Bittner, G. Mönnig, S. Zellerhoff et al., "Randomized study comparing duty-cycled bipolar and unipolar radiofrequency with point-by-point ablation in pulmonary vein isolation," Heart Rhythm, vol. 8, no. 9, pp. 1383-1390, 2011.

[87] A. Bulava, J. Haniš, D. Sitek et al., "Catheter ablation for paroxysmal atrial fibrillation: a randomized comparison between multielectrode catheter and point-by-point ablation," Pacing and Clinical Electrophysiology, vol. 33, no. 9, pp. 1039-1046, 2010.

[88] C. Tivig, L. Dang, H. P. Brunner-La Rocca, S. Özcan, F. Duru, and C. Scharf, "Duty-cycled unipolar/bipolar versus conventional radiofrequency ablation in paroxysmal and persistent atrial fibrillation," International Journal of Cardiology, vol. 157, no. 2, pp. 185-191, 2012.

[89] C. Herrera Siklódy, T. Deneke, M. Hocini et al., "Incidence of asymptomatic intracranial embolic events after pulmonary vein isolation: comparison of different atrial fibrillation ablation technologies in a multicenter study," Journal of the American College of Cardiology, vol. 58, no. 7, pp. 681-688, 2011.

[90] T. Deneke, D. I. Shin, O. Balta et al., "Postablation asymptomatic cerebral lesions: long-term follow-up using magnetic resonance imaging," Heart Rhythm, vol. 8, no. 11, pp. 17051711, 2011.

[91] F. Gaita, J. F. Leclercq, B. Schumacher et al., "Incidence of silent cerebral thromboembolic lesions after atrial fibrillation ablation may change according to technology used: comparison of irrigated radiofrequency, multipolar nonirrigated catheter and cryoballoon," Journal of Cardiovascular Electrophysiology, vol. 22, no. 9, pp. 961-968, 2011. 


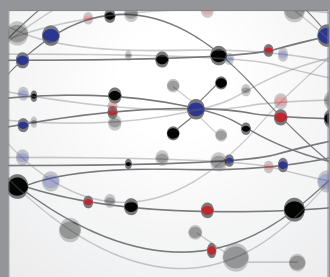

The Scientific World Journal
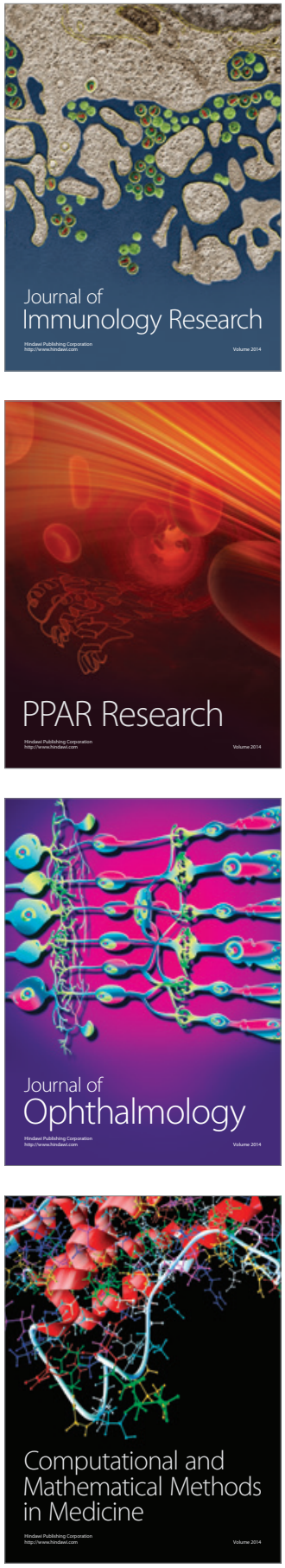

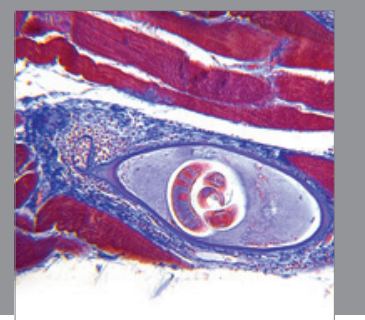

Gastroenterology

Research and Practice
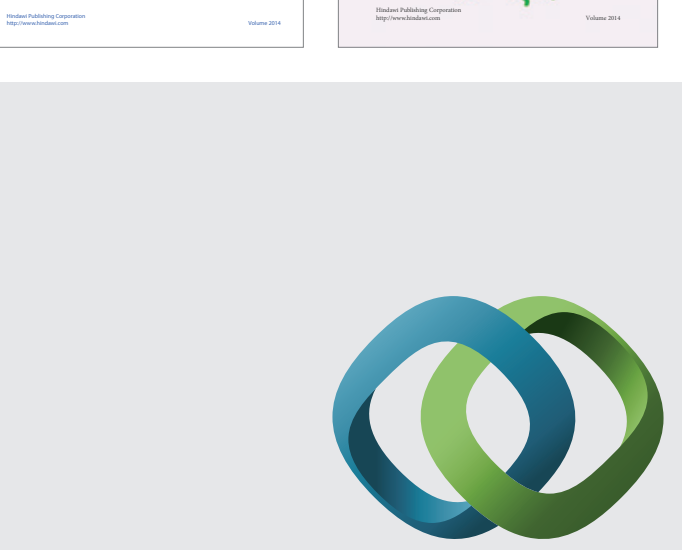

\section{Hindawi}

Submit your manuscripts at

http://www.hindawi.com
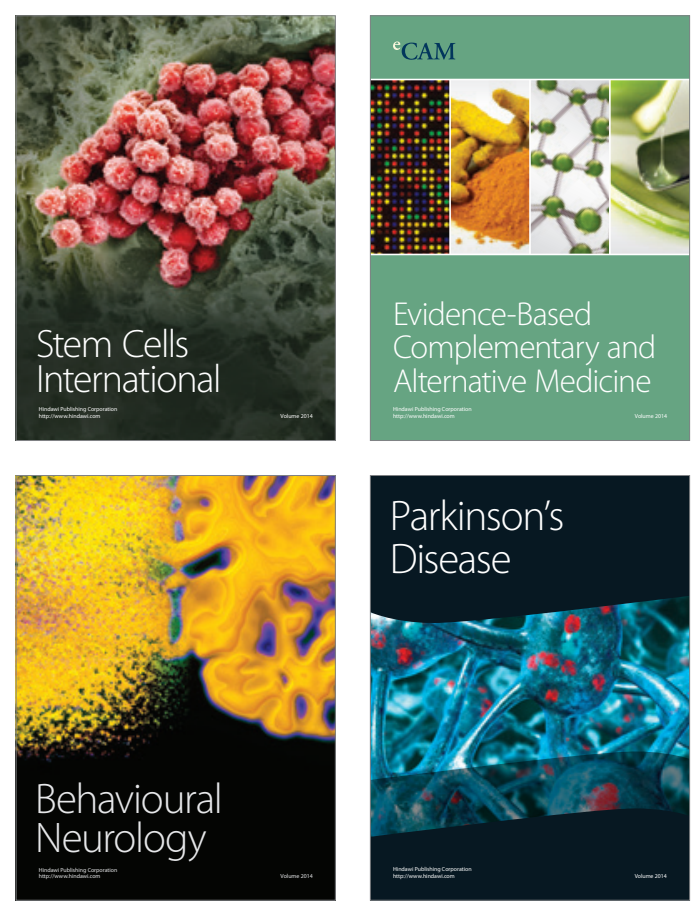

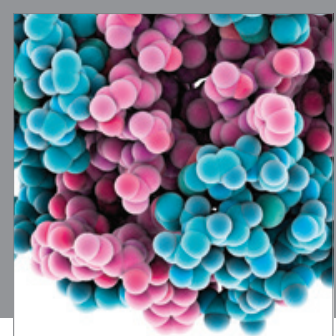

Journal of
Diabetes Research

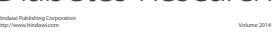

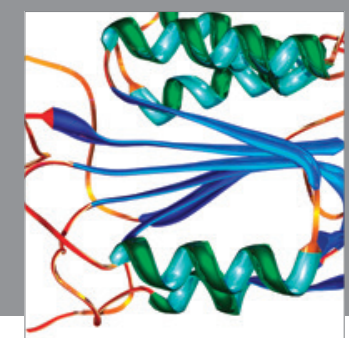

Disease Markers
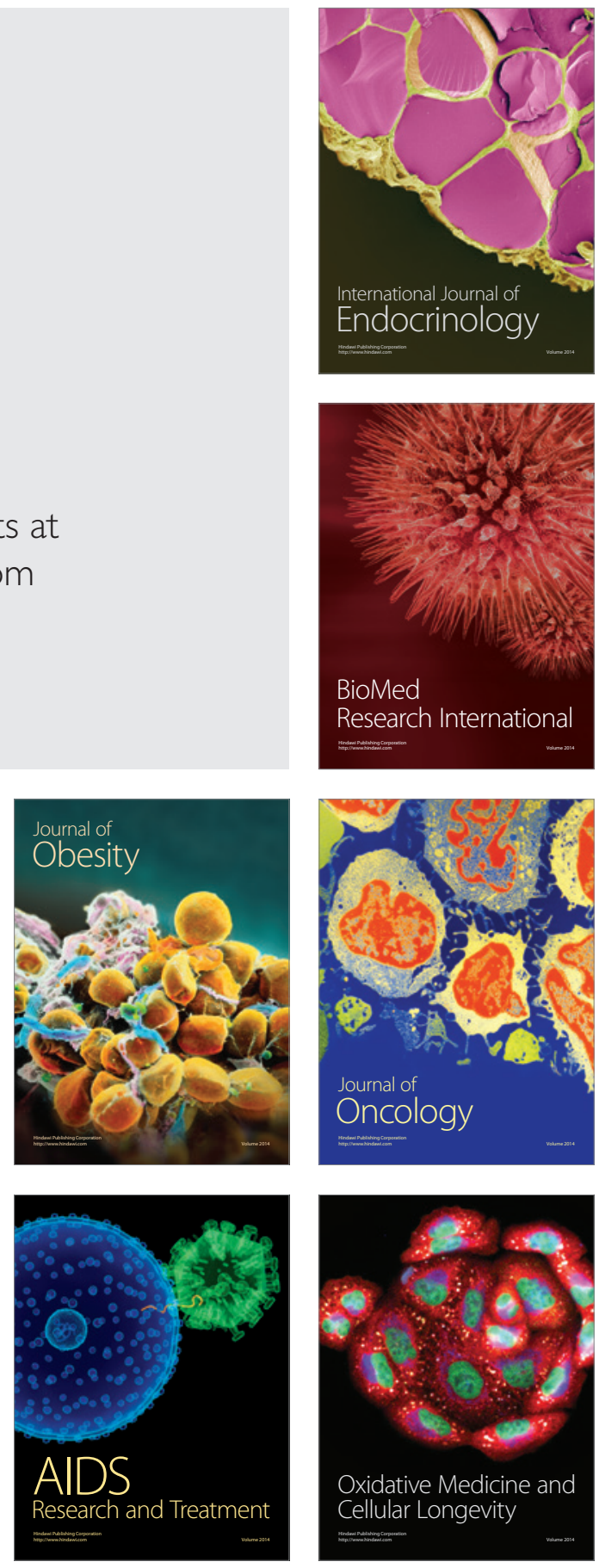